\title{
Análisis de los efectos producidos en el Bienestar Psicológico por motivo de la pandemia Covid-19
}

\section{Analysis of the effects of the COVID-19 pandemic on Psychological Well-being}

1 Julio Alfonso Mocha Bonilla https://orcid.org/0000-0002-8903-4746 Universidad Técnica de Ambato, Facultad de Ciencias Humanas y de la Educación, Carrera de Pedagogía de la Actividad Física y Deporte. Unidad de Investigación FCHE. Ambato, Ecuador ja.mocha@uta.edu.ec

2 Washington Ernesto Castro Acosta https://orcid.org/0000-0001-5028-9680 Universidad Técnica de Ambato, Facultad de Ciencias Humanas y de la Educación, Carrera de Pedagogía de la Actividad Física y Deporte. Unidad de Investigación FCHE. Ambato, Ecuador we.castro@uta.edu.ec

3 Patricio Gustavo Ortiz Ortiz (iD) https://orcid.org/0000-0001-7104-3428 Universidad Técnica de Ambato, Facultad de Ciencias Humanas y de la Educación, Carrera de Pedagogía de la Actividad Física y Deporte. Unidad de Investigación FCHE. Ambato, Ecuador patriciogortizo@uta.edu.ec

4 Raúl Guillermo Zambrano Pontón (iD) https://orcid.org/0000-0002-4009-2726 Universidad Nacional de Chimborazo, UNACH, Carrera de Comunicación Social. Chimborazo, Ecuador gzambrano@unach.edu.ec

Artículo de Investigación Científica y Tecnológica Enviado: 24/12/2021 Revisado: 29/12/2021 Aceptado: 03/01/2022 Publicado:08/03/2023

DOI: https://doi.org/10.33262/concienciadigital.v6i1.4.2025

Cítese: $\quad$ Mocha Bonilla, J. A., Castro Acosta, W. E., Ortiz Ortiz, P. G., \& Zambrano Pontón, R. G. (2023). Análisis de los efectos producidos en el Bienestar Psicológico por motivo de la $\begin{array}{llll}\text { pandemia } & \text { Covid-19. } & \text { ConcienciaDigital, } & 6(1.4),\end{array}$ https://doi.org/10.33262/concienciadigital.v6i1.4.2025

CONCIENCIA DIGITAL, es una Revista Multidisciplinar, Trimestral, que se publicará en soporte electrónico tiene como misión contribuir a la formación de profesionales competentes con visión humanística y crítica que sean capaces de exponer sus resultados investigativos y científicos en la misma medida que se promueva mediante su intervención cambios positivos en la sociedad. https://concienciadigital.org

La revista es editada por la Editorial Ciencia Digital (Editorial de prestigio registrada en la Cámara Ecuatoriana de Libro con No de Afiliación 663) www.celibro.org.ec 


\section{Palabras claves: bienestar psicológico, pandemia, covid-19, depresión.}

Resumen

Introducción. Durante el mes de enero del 2020 la Organización Mundial de la Salud notificó la aparición de un nuevo genoma conocido como sarcov-2, desde allí la población a nivel mundial ha tenido que afrontar la crisis sanitaria por motivo de la pandemia del covid-19, la cual se agudizó durante el año 2021 en Ecuador y la ciudad de Ambato; la problemática de la emergencia sanitaria y el confinamiento repercutió en la salud física de las personas, lo cual generó efectos en la salud mental, las emociones y los sentimientos de los individuos. Objetivo. El presente estudio tuvo como propósito analizar el bienestar psicológico y los efectos producidos por motivo de la pandemia Covid-19 en las familias del barrio la pradera. Metodología. El estudio se realizó durante la pandemia del Covid-19 en varias familias del barrio La Pradera de la ciudad de Ambato, el trabajo investigativo fue desarrollado bajo un enfoque mixto, se aplicó una metodología tipo descriptiva; la muestra de estudio estuvo conformada por 20 sujetos todos cabeza de hogar pertenecientes al barrio residencial la pradera, los datos fueron tomados y analizados en la etapa más fuerte de confinamiento sanitario en la ciudad de Ambato; el instrumento para la recolección de la información presenta categorías correspondientes al bienestar psicológico para adultos (BIEPS-A), el mismo que se divide en cinco categorías: Aceptación de sí mismo, control de situaciones, vínculos sociales, autonomía y proyectos de vida, el cual fue aplicado mediante herramientas tecnológicas para evitar los contagios. Resultados. Los datos revelan que el $95 \%$ de los encuestados poseen un nivel alto de aceptación de sus equivocaciones, es decir, autoconfianza para tomar las mejores decisiones para afrontar sus problemas familiares en la etapa de pandemia, sin embargo, el 50\% de los encuestados tienen dudas en tomar sus decisiones solos, dependen de sus familiares, se pudo observar que el $65 \%$ consideran que los efectos de la pandemia son en la parte económica, un $20 \%$ en la salud, el $10 \%$ en la educación y tan solo el $5 \%$ mencionan la afectación de empleo. Conclusiones. En el barrio la pradera de la ciudad de Ambato se encontró que en la etapa de pandemia sanitaria se necesita total apoyo por parte de la familia para afrontar los aspectos psicológicos y sociales producidos por la pandemia. 
Keywords: psychological well-being, pandemic, covid-19, depression
Abstract

Introduction. During the month of January 2020 the World Health Organization notified the appearance of a new genome known as sarcov-2, since then the population worldwide has had to face the health crisis due to the covid-19 pandemic, which worsened during the year 2021 in Ecuador and the city of Ambato; the problem of the health emergency and the confinement had an impact on the physical health of people, which generated effects on the mental health, emotions and feelings of individuals. Objective. The purpose of this study was to analyze the psychological well-being and the effects produced by the Covid-19 pandemic in the families of the Pradera neighborhood. Methodology. The study was carried out during the Covid-19 pandemic in several families of the Pradera neighborhood in the city of Ambato, the research work was developed under a mixed approach, a descriptive methodology was applied; the study sample consisted of 20 subjects, all heads of household belonging to the residential neighborhood of the Pradera, the data were collected and analyzed in the strongest stage of sanitary confinement in the city of Ambato; the instrument for the collection of information presents categories corresponding to the psychological well-being for adults (BIEPS-A), which is divided into five categories: Self-acceptance, control of situations, social bonds, autonomy and life projects, which was applied by means of technological tools to avoid contagions. Results. The data reveal that $95 \%$ of the respondents have a high level of acceptance of their mistakes, that is, self-confidence to make the best decisions to face their family problems in the pandemic stage, however, $50 \%$ of the respondents have doubts in making their decisions alone, they depend on their relatives, it could be observed that $65 \%$ consider that the effects of the pandemic are in the economic part, $20 \%$ in health, $10 \%$ in education and only $5 \%$ mention the affectation of employment. Conclusions. In the Pradera neighborhood of the city of Ambato, it was found that in the pandemic health stage, total support is needed from the family to face the psychological and social aspects produced by the pandemic. 


\section{Introducción}

El 12 de enero del 2020 la OMS recibió la noticia de la aparición de un genoma secuenciado de un nuevo virus y lo nombró (coronavirus), el 11 de febrero se conoce el nombre oficial de esta enfermedad, que hoy en día de denomina pandemia Covid-19. Según Claros (2011) el bienestar psicológico y sus efectos están relacionado con la salud integral y la calidad de vida, por su parte Solano (2009), afirma que el bienestar psicológico está directamente relacionado con el logro de los objetivos personales, pues incluye los aspectos valorativos y afectivos (Bonilla, 2020). los cuales llevan al individuo hacia la proyección de su futuro de vida (Zapata. 2013), por consiguiente, el bienestar psicológico es una disposición entre las emociones y los sentimientos, permite valorar los componentes sociales y psicológicos relacionados con la satisfacción con la vida relacionados con el bienestar psicológico (Bonilla, 2019). La pandemia provocada por el Covid-19 ha hecho que las familias del barrio la pradera estén enfrentando mayor vulnerabilidad psicosocial en su aspecto emocional, ya que al enfrentar diversos problemas económicos y los efectos del virus el entorno familiar empiezan a presentar algunos síntomas negativos asociados con la depresión, la ansiedad, el estrés y la angustia emocional de tener que enfrentar el contagio o la pérdida de un familiar (López-Jaimes et al., 2020), es decir, efectos y desestabilidad en el bienestar psicológico.

El bienestar psicológico en la pandemia Covid-19 según Becerra Cacho (2021) ha afectado a todo el mundo, desde finales del año 2019 se presentan investigaciones relacionadas con el Bienestar Psicológico durante la emergencia sanitaria; varios estudios relacionan al estrés con el bienestar psicológico para determinar hasta qué punto pueden afectar la salud mental en los individuos; las investigaciones realizadas destacan que el peligro del contagio, las pérdidas económicas y los cambios sociales afectan el bienestar psicológico en las familias. Otros autores como López-Jaimes et al. (2020), afirman que para el año 2020 se presentaron los primeros efectos negativos en la salud mental, tanto en adolescentes como en adultos, varias investigaciones muestran resultados favorables sobre el apoyo social más que en el aspecto de salud; lo que quiere decir es que las personas con matrimonio estables tienen mayor apoyo social (Vivaldi, 2012).

Por su parte Durán (2010), destaca que los trabajadores día a día están enfrentando una gran presión fruto de la pandemia, generando así estrés laboral y familiar; con la aparición del confinamiento a consecuencia del Covid-19, la teórica científica destaca que el bienestar psicológico incorpora concepciones relacionadas con la satisfacción con la vida, afectividad positiva, búsqueda del crecimiento personal, es decir, la capacidad humana de actuar en el presente y futuro de la vida. En definitiva, se pretende conocer el bienestar psicológico de las familias del barrio la pradera con la finalidad de poder afrontar de manera positiva la situación actual que se enfrenta como producto de la pandemia (Rangel, 2010). 
La pandemia ha constituido el rompimiento de la cotidianidad, el sarcov2 nos ha tomado de una manera sorpresiva ya que se está produciendo nuevas variantes del virus, con lo cual se empezó a observar desorden en las emociones y la conducta de las personas. El contexto ecuatoriano no es la excepción y en la ciudad de Ambato se presenta la problemática de ansiedad y depresión en la familia del barrio la pradera por la situación del virus Covid-19 y las nuevas variantes que se están generando, pues las familias están muy preocupadas por la gravedad de la pandemia, muchos padres de familia e hijos que son sustento de hogar a la hora de salir de casa, lo hacen con sentimientos de incompetencia por miedo al contagio. En tal sentido, a cada integrante de la familia le preocupa la situación ya que el sistema de salud está colapsado, situación que se va apoderando de las emociones del individuo y va afectando el bienestar psicológico generando así estrés emocional; problemas como el desempleo, la perdida de trabajo, el no contar con recursos para los estudios de sus hijos, la falta de medicina, y los elevados costos que se debe afrontar cuando una persona se infecta, son factores que están deprimiendo a los miembros de las familias del barrio la pradera en la ciudad de Ambato.

El covid-19 ha generado un fuerte impacto en el bienestar psicológico y la salud mental, la cual es esencial para una buena calidad de vida (Rosales et al., 2020). La salud mental es un concepto muy amplio que requiere un tratamiento social, donde los comportamientos subjetivos de carácter psicológicos están conectados con la salud personal, la finalidad es conocer los indicadores de salud metal y el funcionamiento del ser como regulador y motivador del bienestar (Oramas, 2013). El estrés, la ansiedad y la depresión son aspectos que afectan la salud mental en los individuos (Piqueras, 2008), por lo que se aconseja para una buena salud mental es realizar ejercicio en compañía de un can y reducir los efectos del estrés (Ramírez, 2011), sin embargo, es necesario vincularlo con la incertidumbre de las consecuencias sociales del aislamiento (Velázquez, 2020). El sentimiento de responsabilidad es muy grande porque incluye la importancia de un buen cuidado personal y familiar en cuanto a la prevención del COVID-19. Las personas que están padeciendo de afectaciones producto del contagio están buscando ayuda psicológica y médica para poder combatirlo, por lo cual es necesario hacer una valoración del aspecto emocional especialmente en estos tiempos de emergencia sanitaria, es decir, analizar el componente del bienestar psicológico en las personas.

La Organización Mundial de la Salud en el 2019 presento un informe cuyos datos mostraban que cada 40 segundos una persona se suicidaba por efecto de la angustia o la depresión; estas dos causas están asociadas a los síntomas del estrés y los problemas psicológicos, en primera instancia tenemos al estrés agudo, el cual es fácil de detectar y tratarse, sin embargo, el estrés crónico es mucho más complicado en su tratamiento, actualmente está muy relacionado con la pandemia COVID-19, pues las personas que padecen esta enfermedad están presentando síntomas de depresión, ansiedad e incluso acciones fatales (Dolan, 2020), de aquí el impacto negativo que presenta la pandemia en 
la ciudad de Ambato por los problemas emocional producto del contagio del virus. Ante la problemática existente el estudio tiene como objetivo analizar el bienestar psicológico y los efectos producidos por la pandemia Covid-19 en las familias del barrio la pradera de Ambato, durante los meses de enero a julio 2021.

\section{Metodología}

Para el estudio relacionado con el bienestar psicológico y los efectos producidos por la pandemia Covid-19 en las familias del barrio la pradera en Ambato, durante enero a julio 2021 se presentó un enfoque de investigación de tipo cuali-cuantitativo, también conocido como investigación mixta; fue cuantitativo porque se recogieron y analizaron datos de carácter numérico sobres las categorías correspondientes al bienestar psicológico de muestra de estudio, el mismo que se divide en 5 categorías: Aceptación de sí mismo, control de situaciones, vínculos sociales, autonomía y proyectos de vida; fue cualitativa porque se registraron datos mediante una escala tipo Likert distribuida en tres nivel: de acuerdo (alto), ni de acuerdo ni en desacuerdo (medio) y en desacuerdo (bajo), se utilizó la escala de Likert porque es una escala de calificación ideal para medir reacciones, actitudes y los comportamientos de una persona, toma el nombre de escala Likert en honor al psicólogo Rensis Likert.

La muestra corresponde a las familias del barrio la pradera de la ciudad de Ambato, la cual se obtuvo mediante un muestreo aleatorio 20 integrantes cabeza de hogar de varias familias, las cuales están ubicadas en el barrio la pradera de la ciudad de Ambato, familias cercanas al lugar de residencia de los investigadores.

En primera instancia el estudio de caso utilizó la investigación bibliográfica o también llamada documental, mediante la cual se exploró la problemática y los antecedentes de investigación revisando la literatura científica para conocer el bienestar psicológico y los efectos que se está produciendo por el motivo de la pandemia COVID-19 en las familias del barrio la pradera de la ciudad de Ambato.

Posteriormente se aplicó una investigación descriptiva porque en los resultados se describen las características de la muestra de estudio (familias del barrio la pradera de Ambato-Ecuador), lo cual nos ha ayudado en la recolección de la información referente al bienestar psicológico y los efectos producidos por la pandemia Covid-19. Por otra parte, se realizó una investigación explicativa para dar a conocer el fenómeno objeto de estudio en nuestro caso explicar concordamos con Eidman et al. (2020), al estudiar los efectos que se está produciendo por la actual pandemia en el bienestar psicológico, emocional y social de las familias.

Para la recolección de la información se aplicó una encuesta validada por la comunidad científica ya que el bienestar psicológico adquiere un estudio objetivo en adolescentes y 
adultos (Lara, 2014). La escala de Bienestar Psicológico para Adultos (BIEPS-A) presenta una evaluación rápida y sencilla basada en 5 tipos de categorías, las cual contiene 13 preguntas relacionadas con el bienestar emocional y psicológico de la persona; el cuestionario fue aplicado de manera online para contribuir con el cuidado personal y evitar el contagio del COVID-19, el formulario fue diseñado en la plataforma de Google forms para contribuir con las normas de bioseguridad.

Los principales resultados en las cinco categorías de estudio se muestran a continuación:

\section{Resultados}

Una vez realizada la respectiva encuesta online referente al tema bienestar psicológico y los efectos producidos por la pandemia Covid-19 en las familias del barrio la pradera en Ambato, durante el mes de enero a julio 2021 se obtienen los siguientes resultados:

Categoría 1. Puedo aceptar mis equivocaciones y tratar de mejorar. Esta pregunta corresponde a la categoría de aceptación, los principales resultados se observan en la Tabla. 1. En la cual se evidencia que 19 personas correspondiente al $95 \%$ de los encuestados poseen un nivel alto de aceptación de sus equivocaciones, es decir, autoconfianza para tomar las mejores decisiones en la etapa de pandemia y durante la emergencia sanitaria.

\section{Tabla 1}

Categoría: Aceptación de sí mismo

\begin{tabular}{lc}
\hline Categorías & $\begin{array}{c}\text { Pre. 11. Puedo aceptar mis } \\
\text { equivocaciones y tratar de mejorar. }\end{array}$ \\
\hline 1. De acuerdo (ALTO) & 19 \\
2. Ni de acuerdo ni en & \\
desacuerdo (MEDIO) & 1 \\
3. En desacuerdo (BAJO) & 0 \\
\hline
\end{tabular}

Categoría 2. Pregunta 2. Si algo me sale mal puedo aceptarlo, admitirlo; Pregunta 13. Encaro sin mayores problemas mis obligaciones diarias. Estas preguntas corresponden a la categoría de control de situaciones en los sujetos de estudio, la tabla 2 muestra los resultados del barrio la pradera de la cuidad de Ambato.

En la tabla 2. En base a los resultados de la pregunta 2 y 13 se observa mayor índice de aceptación, es decir 17 de 20 personas, se puede concluir que la gran mayoría de las personas encuestadas puede afrontar sus problemas familiares durante la pandemia. 
Tabla 2

Categoría: Control de situaciones

\begin{tabular}{lcc}
\hline & $\begin{array}{l}\text { Pre. 2. Si algo me sale mal } \\
\text { puedo aceptarlo, admitirlo. }\end{array}$ & $\begin{array}{l}\text { Pre. 13. Encaro sin mayores } \\
\text { problemas mis obligaciones diarias. }\end{array}$ \\
\hline $\begin{array}{l}\text { Categorías } \\
\begin{array}{l}\text { 2. De acuerdo (ALTO) } \\
\text { desacuerdo (MEDIO) }\end{array}\end{array}$ & 15 & 17 \\
$\begin{array}{l}\text { 3. En desacuerdo } \\
\text { (BAJO) }\end{array}$ & 3 & 2 \\
\hline
\end{tabular}

Categoría 3. Pregunta 4. Puedo decir lo que pienso sin mayores problemas; Pregunta 9. En general hago lo que quiero, soy poco influenciable; Pregunta 12. Puedo tomar decisiones sin dudar mucho. Estas preguntas corresponden a la categoría Autonomía presente en la tabla. 3.

Los resultados de las preguntas 4, 9 y 12 que corresponde a la categoría de autonomía; de un total de 20 personas encuestadas los datos muestran que la mitad de las personas encuestadas tienen dudas en tomar sus decisiones por su propia cuenta, es decir, dependen de otras personas en este caso de sus familias.

Tabla 3

Categoría: Autonomía

\begin{tabular}{lccc}
\hline & $\begin{array}{l}\text { Pre. 4. Puedo decir lo } \\
\text { que pienso sin } \\
\text { mayores } \\
\text { problemas. }\end{array}$ & $\begin{array}{l}\text { Pre. 9. En general hago } \\
\text { lo que quiero, soy poco } \\
\text { influenciable. }\end{array}$ & $\begin{array}{l}\text { Pre. 12. Puedo tomar } \\
\text { decisiones sin dudar } \\
\text { mucho. }\end{array}$ \\
\hline $\begin{array}{lccc}\text { 1. De acuerdo (ALTO) } \\
\begin{array}{l}\text { 2. Ni de acuerdo ni en } \\
\text { desacuerdo (MEDIO) }\end{array}\end{array}$ & 15 & 9 & 10 \\
\hline 3. En desacuerdo (BAJO) & 3 & 10 & 9 \\
\hline
\end{tabular}

Categoría 4. Pregunta 5. Generalmente le caigo bien a la gente; Pregunta 7. Cuento con personas que me ayudan si lo necesito; Pregunta 8. Creo que en general me llevo bien con la gente; Estas preguntas corresponden a la categoría vínculos psicosociales aplicada a los familiares del barrio la pradera de la ciudad de Ambato; en la tabla 4 se puede apreciar los principales resultados correspondientes a las preguntas 5, 7 y 8 que corresponde a la categoría vínculos psicosociales; de las 20 personas encuestadas, nos lleva a concluir que los jefes de hogar de las familias de barrio la pradera en los momentos de pandemia necesitan el apoyo emocional y psicológico de sus familiares. 


\section{Tabla 4}

Categoría: Vínculos Psicosociales

\begin{tabular}{lccc} 
& $\begin{array}{l}\text { Pre. 5. Generalmente } \\
\text { le caigo bien a la } \\
\text { gente. }\end{array}$ & $\begin{array}{l}\text { Pre. 7. Cuento con } \\
\text { personas que me ayudan } \\
\text { si lo necesito. }\end{array}$ & $\begin{array}{l}\text { Pre. 8. Creo que en } \\
\text { general me llevo bien } \\
\text { con la gente. }\end{array}$ \\
\hline $\begin{array}{l}\text { 1. De acuerdo (ALTO) } \\
\text { 2. Ni de acuerdo ni en }\end{array}$ & 7 & 18 & 14 \\
desacuerdo (MEDIO) & 9 & 1 & 6 \\
3. En desacuerdo (BAJO) & 4 & 1 & 0 \\
\hline
\end{tabular}

Categoría 5. Pregunta 1. Creo que sé lo que quiero hacer con mi vida; Pregunta 3. Me importa pensar qué haré en el futuro; Pregunta 6. Siento que podré lograr las metas que me proponga; Pregunta 10. Soy una persona capaz de pensar en un proyecto para mi vida. Estas preguntas corresponden a la categoría proyectos con la vida, la tabla 5 muestra los respectivos resultados. En función de la pregunta 1, 3, 6 y 10 que corresponde a la categoría proyectos para la vida; de las 20 personas encuestadas las 17 personas presentan un nivel alto, es decir, se proponer un proyecto de vida a futuro a pesar de las problemáticas de la pandemia.

\section{Tabla 5}

Categoría: Proyectos para la vida

\begin{tabular}{|c|c|c|c|c|}
\hline Categorías & $\begin{array}{l}\text { Pre. 1. Creo que } \\
\text { sé lo que quiero } \\
\text { hacer con mi } \\
\text { vida. }\end{array}$ & $\begin{array}{l}\text { Pre. 3. Me } \\
\text { importa pensar } \\
\text { qué haré en el } \\
\text { futuro. }\end{array}$ & $\begin{array}{l}\text { Pre. 6. Siento que } \\
\text { podré lograr las } \\
\text { metas que me } \\
\text { proponga. }\end{array}$ & $\begin{array}{l}\text { Pre. } 10 \text {. Soy una } \\
\text { persona capaz de } \\
\text { pensar en un } \\
\text { proyecto para mi } \\
\text { vida }\end{array}$ \\
\hline 1. De acuerdo (ALTO) & 16 & 19 & 19 & 17 \\
\hline $\begin{array}{l}\text { 2. Ni de acuerdo ni en } \\
\text { desacuerdo (MEDIO) }\end{array}$ & 4 & 1 & 1 & 3 \\
\hline 3. En desacuerdo (BAJO) & 0 & 0 & 0 & 0 \\
\hline
\end{tabular}

Finalmente, en la Figura 1 luego de aplicar la encuesta online a los correspondientes 20 sujetos de estudio se puede observar los resultados de los efectos producidos por la pandemia Covid-19 en los integrantes de las familias del barrio la pradera de AmbatoEcuador. En la figura 1. Relacionada con los efectos de la pandemia en el bienestar psicológico, del total de encuestados se puede observar que el $65 \%$ consideran que los 
efectos son en la parte económica, un $20 \%$ en la salud, el $10 \%$ en educación y tan solo el $5 \%$ afecta al empleo.

\section{Figura 1}

\section{Categoría: Efectos de la pandemia en el bienestar psicológico}

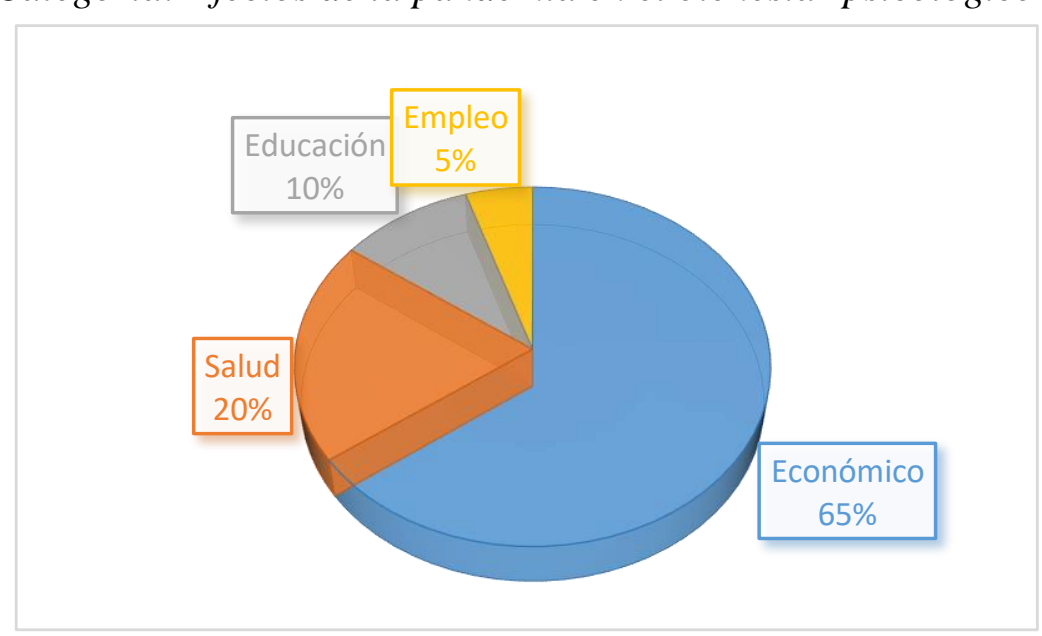

\section{Conclusiones}

- En relación con el objetivo del estudio se pudo realizar un análisis descriptivo sobre el bienestar psicológico de 20 integrantes del barrio la pradera de la ciudad de Ambato encontrando que en la etapa de pandemia sanitaria por el Covid-19 se necesita total apoyo por parte de la familia para afrontar los aspectos psicológicos y sociales.

- En relación con la escala para analizar el bienestar psicológico se presentan 5 categorías, los resultados encontrados muestran que el $95 \%$ de encuestados en la categoría aceptación aceptan sus equivocaciones tratando de mejorar sus errores, es decir, poseen autoconfianza, en la categoría control de situaciones se puede destacar un buen nivel de bienestar personal, es decir, buenas relaciones interpersonal en el aspecto afectivo y emocional, en la categoría autonomía el 50 $\%$ los encuestados presentan dudas al momento de tomar decisiones de manera personal, es decir, necesitan el apoyo de sus familiares cercanos, lo cual concuerda con la categoría vínculos sociales donde se aprecia que la gran mayoría de los encuestados sienten el apoyo del entorno familiar, por ende existe buenas relaciones sociales y familiares, mientras que en la categoría proyectos para la vida el $95 \%$ se proponen metas a futuro a pesar de las adversidades de puedan generar la pandemia.

- Finalmente se puede observar claramente que los mayores efectos producidos por motivo de la actual pandemia y la emergencia sanitaria, se puede observar las afectaciones en el aspecto económico, seguido de la situación de salud, mientras 
que en el factor educación y empleo se presentan valores muy bajos, se asume estas afirmaciones por la actual educación virtual y el teletrabajo de la muestra de estudio.

\section{Referencias Bibliográficas}

Becerra Cacho, L. S. (2021). La pandemia Covid-19 y su impacto en el bienestar psicológico. Cajamarca - Perú: Universidad Privada Antonio Guillermo.

Bonilla, J. A. M., Benítez, M. C., \& Galak, E. L. (2020). Apoyo motivacional docente y necesidades psicológicas básicas en la Educación Física: una revisión sistemática. Ciencia Digital, 4(1.1.), 5-20.

Bonilla, J. A. M., Ricardo, Y. R., Fuente, C. D. C., \& López, X. C. M. (2019). La autoestima, la autonomía y el apoyo a las necesidades psicológicas básicas en personas con discapacidad visual. Ciencia Digital, 3(2.5), 120-133.

Claros, J. A. (2011). Actividad física: estrategia de promoción de la salud. Hacia la Promoción de la Salud, 16(1), 202-218.

Dolan, S. \&. (2020). Covid-19, Estrés, Autoestima, Valores y bienestar psicológico: ¿Cómo evaluar los riesgos de estar deprimido, ansioso o, incluso de suicidarse? The European Business Review, 1-16. The European Business Review, 1-16.

Durán, M. M. (2010). Bienestar psicológico: el estrés y la calidad de vida en el contexto laboral. Revista nacional de administración, 1(1), 71-84.

Eidman, L., Bender, V., Arbizu, J., Lamboglia, A. T., \& Valle, L. C. D. (2020). Bienestar emocional, psicológico y social en adultos argentinos en contexto de pandemia por covid-19. Psychologia. Avances de la Disciplina, 14(2), 69-80.

Lara, S. A. (2014). Análisis Psicométrico de la Escala de Bienestar Psicológico para Adultos en estudiantes universitarios de Lima: un enfoque de ecuaciones estructurales. Psychologia. Avances de la disciplina, 8(1), 23-31.

López-Jaimes, R. J., Medina-Ortiz, O., Mendoza-Rincón, B. M., Arenas-Villamizar, V. V., Araque-Castellanos, F., Jaimes-Parada, G. Y. \& Cotrino, J. (2020). Bienestar psicológico en mujeres víctimas del conflicto armado durante el confinamiento en pandemia por covid-19. Archivos Venezolanos de Farmacologia y Terapéutica, 39(8), 1026-1035.

Oramas Viera, A. (2013). El bienestar psicológico, un indicador positivo de la salud mental. Cuba: Universidad de las Tunas. 
Piqueras Rodríguez, J. A. (2008). Ansiedad, depresión y salud. Suma psicol, 43-74.

Ramírez, M. T. (2011). Diferencias en estrés percibido, salud mental y física de acuerdo al tipo de relación humano-perro. Revista Colombiana de Psicología, 20(1), 7586.

Rangel, J. V. (2010). El estudio del bienestar psicológico subjetivo. Una breve revisión teórica. Educere, 14(49), 265-275.

Rosales, Y., Mocha-Bonilla, J., \& Ferreira, J. P. (2020). Síndrome de Burnout en estudiantes de la Universidad Técnica de Ambato, Ecuador. Enfermería Investiga, 5(2), 37-41.

Solano, A. C. (2009). El bienestar psicológico: cuatro décadas de progreso. Revista interuniversitaria de formación del profesorado, 23(3), 43-72.

Velázquez, T. (10 de mayo de 2020). Salud mental comunitaria en tiempos del Coronavirus: alcances $y$ desafíos. Obtenido de https://idehpucp.pucp.edu.pe/revista-memoria/articulo/salud-mentalcomunitaria-en-tiempos-del-coronavirus-alcances-y-desafios/

Vivaldi, F. (2012). Bienestar psicológico, apoyo social percibido y percepción de salud en adultos mayores. Terapia psicológica, 30(2), 23-29.

Zapata, A. B. (2013). Evaluación del bienestar psicológico y estrategias de afrontamiento en padres con niños o adolescentes con retraso mental leve. Psiencia: Revista Latinoamericana de Ciencia Psicológica, 5(1), 15-23.

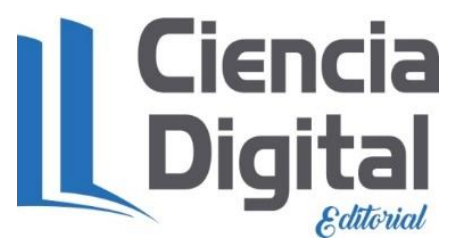


El artículo que se publica es de exclusiva responsabilidad de los autores y no necesariamente reflejan el pensamiento de la Revista Conciencia Digital.

\section{Ciencia \\ LDigital}

El artículo queda en propiedad de la revista y, por tanto, su publicación parcial y/o total en otro medio tiene que ser autorizado por el director de la Revista Conciencia Digital.
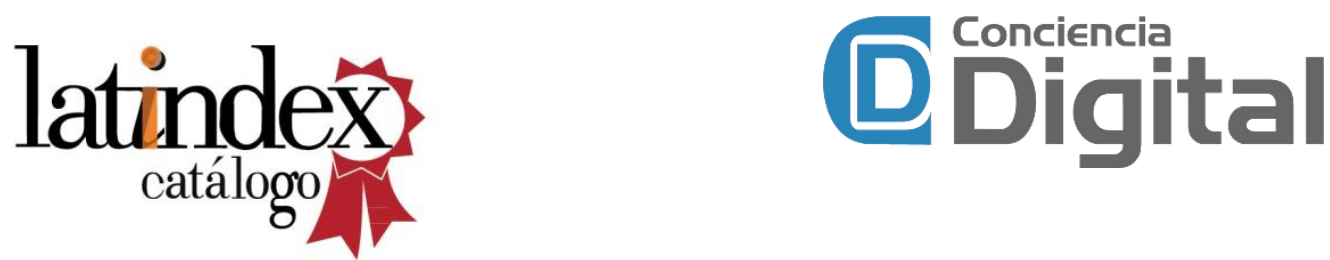

Indexaciones

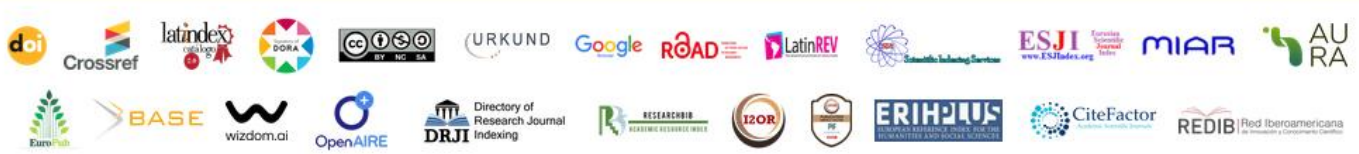

